\title{
Characterisation of a blowfly male-specific neuron using behaviourally generated visual stimuli
}

\author{
Christine Trischler • Norbert Boeddeker • \\ Martin Egelhaaf
}

Received: 21 November 2006 / Revised: 18 January 2007 / Accepted: 19 January 2007

(C) Springer-Verlag 2007

\begin{abstract}
The pursuit system controlling chasing behaviour in male blowflies has to cope with extremely fast and dynamically changing visual input. An identified male-specific visual neuron called Male Lobula Giant 1 (MLG1) is presumably one major element of this pursuit system. Previous behavioural and modelling analyses have indicated that angular target size, retinal target position and target velocity are relevant input variables of the pursuit system. To investigate whether MLG1 specifically represents any of these visual parameters we obtained in vivo intracellular recordings while replaying optical stimuli that simulate the visual signals received by a male fly during chasing manoeuvres. On the basis of these naturalistic stimuli we find that MLG1 shows distinct direction sensitivity and is depolarised if the target motion contains an upward component. The responses of MLG1 are jointly determined by the retinal position, the speed and direction, and the duration of target motimotion. Coherence analysis reveals that although retinal target size and position are in some way inherent in the responses of MLG1, we find no confirmation of the hypothesis that MLG1 encodes any of these parameters exclusively.
\end{abstract}

Keywords Fly · Vision - Chasing behaviour . Naturalistic stimuli $\cdot$ Male-specific neuron

C. Trischler $(\varangle) \cdot$ M. Egelhaaf

Department of Neurobiology, Bielefeld University,

Post Box 100131, 33501 Bielefeld, Germany

e-mail: christine.trischler@uni-bielefeld.de

\section{N. Boeddeker}

Centre for Visual Sciences, RSBS,

Australian National University,

Canberra, ACT 2601, Australia
Abbreviations
HS Horizontal system
MLG1 Male lobula giant (neuron) 1
MLGs Male lobula giant (neurons)
SNR Signal-to-noise ratio
STMD Small target motion detector
VS Vertical system

\section{Introduction}

Males of several fly species pursue potential mates or rivals in visually mediated high-speed aerobatic chases during their courtship behaviour (e.g. Land and Collett 1974; Collett and Land 1975; Wehrhahn 1979; Collett 1980; Wehrhahn et al. 1982; Zeil 1983; Wagner 1986a, b, c; Land 1993a, b). To catch a female fly the male fly has to detect the potential mate and then follow it by adjusting his speed and direction of locomotion to the course of the target. Eventually-if successful-the chase will culminate in a catch of the target and-if the target turns out to be a female conspecific-in copulation (Wehrhahn 1979; Wagner 1986a, b). The chasing behaviour of male flies belongs to the fastest visually controlled behaviours that can be found in nature. How does the underlying neuronal substrate of the visual pathway in the fly's brain achieve both pursuit control and successful capture of conspecific females which are both mandatory prerequisites for mating? Behavioural experiments with male blowflies chasing each other have led to phenomenological models of the control system underlying chasing behaviour (Land and Collett 1974). According to this model the visual cues used for chasing control are the target position on the retina of the pursuer and the retinal target velocity. 
During aerial pursuit the chasing fly minimizes deviations of the target's retinal position from the frontal midline by yaw rotations. Land and Collett's model was extended when it became clear that dummy targets of different sizes are followed in a systematically different way (Boeddeker et al. 2003). To this end an extra visual pathway where the retinal target size controls the forward velocity of the pursuer was added. In numerical simulations of chasing behaviour the proposed control system generates qualitatively the same behaviour as real blowflies (Boeddeker and Egelhaaf 2003, 2005; Hüls 2005). It is one goal of this study to find out whether this model is implemented at the neuronal level in the visual system of male flies.

Our hypothesis is that the specific visual parameters used by the model are separately encoded in different male specific neurons. We test this hypothesis for one of the 12 types of large male-specific visual interneurons (MLG, Male Lobula Giant neuron) that have been identified in the lobula of Calliphora and Sarcophaga (Gilbert and Strausfeld 1991; Strausfeld 1991). These male-specific neurons (MLGs) are most likely the neuronal substrate mediating chasing behaviour (e.g. Hausen and Strausfeld 1980; Gilbert and Strausfeld 1991; Wachenfeld 1994). MLGs terminate on descending neurons, which descend to the thoracic ganglia and have connections with neck and flight motor neurons (Gronenberg and Strausfeld 1991). Regarding physiology, the MLG1 neuron is best investigated so far: it exhibits pronounced direction selectivity, its receptive field subtends the area of the acute zone, and it responds well to small moving objects (Gilbert and Strausfeld 1991; Wachenfeld 1994). Anatomical and physiological characteristics resembling those of MLGs are also described for neurons (STMD, Small Target Motion Detector) found in the lobula of the male hoverfly Eristalis (Nordström et al. 2006).

Other sexual dimorphisms at several levels of the visual system seem to be uniquely adapted to chasing behaviour and are believed to increase the male's abilities to catch females. In male calliphorid flies the medulla, which is the second neuropile along the visual pathway, has more columns than in females, and the lobula and lobula plate (i.e. the third optic neuropile) are larger in males than in females (Strausfeld 1991). During pursuit male flies fixate their target in the frontal region of the retina (e.g. Land and Collett 1974; Wehrhahn 1979; Collett 1980; Zeil 1983; Wagner 1986b; Land 1993a, b; Boeddeker et al 2003). This eye region has been called 'acute zone' and has structural and physiological characteristics that provide the photoreceptors in the acute zone with high spatial resolution and fast and reliable responses (Franceschini et al.
1981; Hardie et al. 1981; Land and Eckert 1985; Land 1997; Hornstein et al. 2000; Burton and Laughlin 2003). The receptive fields of MLG1 and nine other MLG neurons cover the acute zone.

Recent studies indicate that taking the natural stimulus conditions into account can be essential for an understanding of the functional relevance of neuronal computations (e.g. van Hateren 1997; Reinagel 2001; Simoncelli and Olshausen 2001; Burton and Laughlin 2003; Simoncelli 2003; Kayser et al. 2004; Boeddeker et al. 2005; Kern et al. 2005; van Hateren et al. 2005). To this end we combined behavioural and electrophysiological experiments. This allowed us to use dynamical stimuli, as they are experienced by the male fly during chases to analyse the characteristics of a blowfly male-specific neuron for chasing behaviour.

To obtain naturalistic stimuli we recorded with high speed cameras chases after dummy targets moving on circular trajectories. The retinal size and the angular position of the target were reconstructed (Boeddeker et al. 2003) and replayed to male flies, while recording the electrical activity of MLG1. To facilitate comparison of our results to those obtained in previous electrophysiological studies we additionally recorded the responses of MLG1 to experimenter-defined stimuli. Thus, the main goals of this study are (1) to characterise the response properties of MLG1 on the basis of naturalistic visual stimuli and (2) to test whether specific stimulus parameters are encoded by MLG1.

\section{Materials and methods}

\section{Preparation}

The electrophysiological experiments were performed on $12 \mathrm{~h}$-2-day-old male blowflies (Calliphora vicina). Before dissection, the animals were briefly anesthetised with $\mathrm{CO}_{2}$ and mounted ventral side up on a small glass plate by applying wax to abdomen and the wings. The head was tilted forward and fixed with wax to the thorax. Legs and antennas were removed and the wounds were sealed with wax to prevent the animal from drying-up. The head capsule was opened from behind; air sacs and fat tissue were removed. To prevent movements of the brain caused by the peristaltic movement of the gut the proboscis was cut away and the gut was pulled out and fixed to the thorax with wax. The fly's head was aligned with reference to the symmetry of the deep pseudopupil (Franceschini and Kirschfeld 1971). For the electrophysiological experiment the fly was mounted onto a heavy recording table, facing the monitor. 


\section{Electrophysiology}

We recorded electrical responses from the axon or from the primary dendrite of the MLG1 in the right half of the brain. The ground electrode, a blunt glass electrode was filled with Ringer's solution (containing in mM: $\mathrm{NaCl} 128.3, \mathrm{KCl} 5.4, \mathrm{CaCl} 2$ 1.9, $\mathrm{NaHCO} 34.8$, Na2HPO4 3.3, KH2PO4 3.4, glucose 13.9, pH 7.0; all chemicals were from Merck, Darmstadt, Germany) and placed on the left half of the brain. To keep the tissue moist Ringer's solution was supplied to the brain by a syringe connected to the ground electrode holder. For intracellular recording glass capillaries (GC100TF-10; Clark electromedical instruments, Pangbourne, UK; outer diameter $1 \mathrm{~mm}$ ) were pulled on a Flaming/Brown Micropipette Puller (Model P-97, Sutter Instrument Company, Novato, CA). The tip of the recording electrode was filled either with a saturated solution of the fluorescent dye 6-carboxy-fluorescein (Molecular Probes, Eugene, OR) dissolved in $1 \mathrm{M}$ potassium acetate, or with a $12 \mathrm{mM}$ solution of Alexa 488 hydrazide (Molecular Probes) made as follows: $1 \mathrm{mg}$ Alexa 488 was dissolved in $60 \mu \mathrm{l} 5 \mathrm{mM}$ $\mathrm{KOH}$; subsequently $20 \mu \mathrm{l}$ of this Alexa/KOH solution were dissolved in $30 \mu \mathrm{l} 0.25 \mathrm{M} \mathrm{KCl}$ giving the $12 \mathrm{mM}$ Alexa solution. The shaft of the electrode was filled in the latter case $($ Alexa $\mathrm{KOH} / \mathrm{KCl})$ with $0.25 \mathrm{M} \mathrm{KCl}$ and otherwise (6-carboxy-fluorescein) with $0.2 \mathrm{M}$ potassium acetate. The electrodes had resistances between 40 and $120 \mathrm{M} \Omega$. The recorded signals were filtered (low-pass: $2 \mathrm{kHz}$ ), amplified by the use of standard electrophysiological equipment, and fed into a computer through the analogue input of an I/O-card (DT 3001, Data Translation) at a rate of $4 \mathrm{kHz}$. For identification the neuron was iontophoretically filled with fluorescent dye by applying a negative current of 1$2 \mathrm{nA}$ to the recording electrode. After the experiment, the fly's brain (whole mount) was viewed through a fluorescence microscope (Leitz, Wetzlar, Germany) and the filled cell was photographed in vivo with a digital camera. Only cells, which could be unambiguously identified in the whole mount preparation on the basis of prior detailed neuroanatomical studies (Gilbert and Strausfeld 1991; Strausfeld 1991; Wachenfeld 1994), were used for further analysis.

\section{High-speed video analysis of chasing behaviour}

Chasing behaviour was analysed, on the one hand, to characterise the visual stimuli experienced by the chasing fly during aerial pursuit and, on the other hand, to use a selection of such sequences for visual stimulation in electrophysiological experiments.
Male blowflies (age: 2-7 day) were released in a cylindrical flight arena $(91 \times 50 \mathrm{~cm})$. Black painted glass spheres (diameter: 8.3 and $13 \mathrm{~mm}$ ) served as dummy flies and were moved counter-clockwise on a circular track (radius: $10 \mathrm{~cm}$ ) in a horizontal plane. The speed of the spheres ranged between 1 and $1.5 \mathrm{~m} / \mathrm{s}$, which resides within the speed range of real flies. The side walls of the arena consisted of a white drapery, the ceiling and the bottom were homogeneously white, and the arena was illuminated from outside by nine $50 \mathrm{~W}$ halogen lamps. The temperature ranged between 25 and $35^{\circ} \mathrm{C}$. Male flies either chasing the dummy or a real fly were filmed with two orthogonally arranged high speed cameras (MotionPro, Redlake, San Diego, CA. Sampling rate: $500 \mathrm{~Hz}$; spatial resolution: $1024 \times$ 1024 pixels). One camera was positioned besides and the other below the arena, viewing the centre of the arena through holes of the wall. Video sequences were stored as uncompressed 8-bit AVI-files on computer hard disk for off-line processing. The 2-D position and longitudinal body axis orientation of the objects of were determined frame by frame with the aid of custom-built software, using standard image processing algorithms (Lindemann et al. 2003). Knowing the relative position of the two cameras, it is possible to transform 2-D image coordinates into an orthographic 3-D coordinate system (e.g. Zeil 1983; Boeddeker et al. 2003). We determined for each frame of the video the 3-D position and the yaw orientation of the chasing male fly and the position of the target. Furthermore, we estimated in every frame the fly's horizontal gaze direction from its yaw body orientation. Although blowflies can move their head (Land 1973; Hengstenberg 1993), rotations of the head relative to the surroundings about the pitch and roll axes are generally small during spontaneous flight (Schilstra and van Hateren 1998) and yaw head rotations are usually in phase, though somewhat faster than yaw body rotations (van Hateren and Schilstra 1999). Since we could not resolve the head movements in our behavioural data, we assumed the same type of head-body coordination in chasing behaviour as characterised during spontaneous flight. Therefore, we did not simulate these rotational degrees of freedom, but set both the roll and pitch orientation of the chasing fly to $0^{\circ}$ and assumed that the yaw angle of the head was aligned with the body long axis. The reconstruction of the 3-D-trajectories and all further data processing was done in MATLAB.

\section{Visual stimulation}

The position of stimuli is given by the coordinates $\psi$ and $\theta$, denoting the horizontal and vertical angular 
positions of the stimulus-centre with respect to the longitudinal axis of the head (Fig. 1a). $\psi=0^{\circ}$ and $\theta=0^{\circ}$ are defined by the cross-sections of the horizontal eye equator with the vertical symmetry plane of the eye. Positions $\theta>0^{\circ}$ and $\theta<0^{\circ}$ are in the dorsal and ventral visual hemisphere, respectively; positions $\psi>0^{\circ}$ and $\psi<0^{\circ}$ are in the right and left hemisphere of the visual field, respectively.

For visual stimulation, two types of stimuli were displayed: experimenter-defined ('artificial') stimuli and behaviourally generated ('naturalistic') stimuli. Stimuli were presented on a monitor screen (Tektronix 608, Tektronix, Wilsonville, OR), which was positioned in front of the fly covering the fly's visual field from $\theta=-10^{\circ}$ to $\theta=60^{\circ}$ vertically and from $\psi=-40^{\circ}$ to $\psi=40^{\circ}$ horizontally.

Artificial stimuli consisted of a square dot or a bar moving with a constant velocity of $180^{\circ} / \mathrm{s}$. Dots sized $5^{\circ}$ $\times 5^{\circ}$ moved either upward or downward between
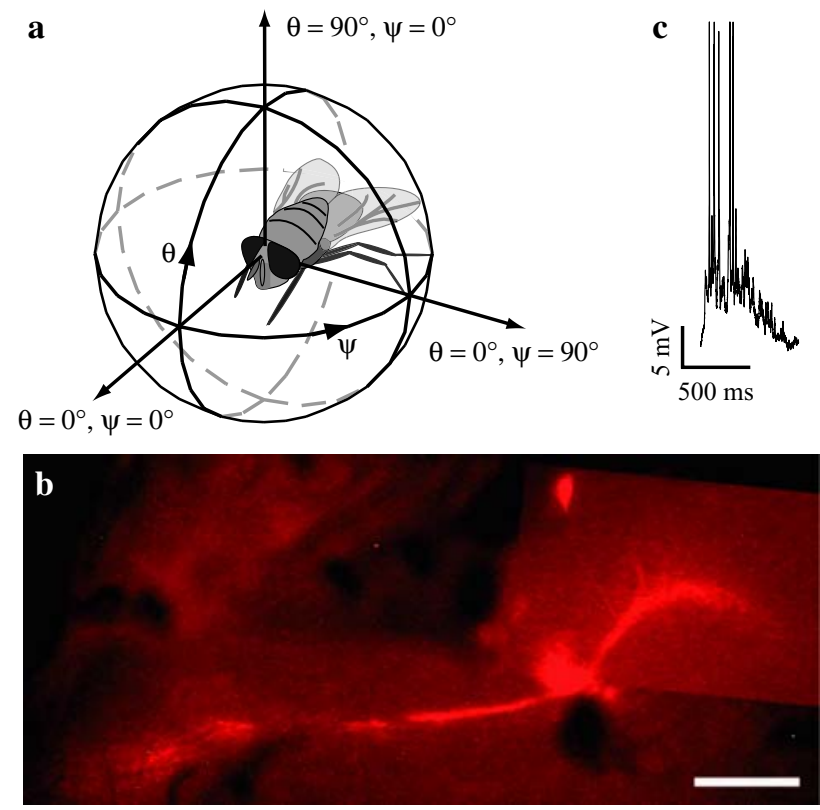

Fig. 1 Polar coordinate system of the fly's visual field and the anatomy and electrical responses of MLG1. a A particular position in the visual field of the fly is specified by the angles of azimuth $\psi$ and elevation $\theta$. The position $\psi=0^{\circ}, \theta=0^{\circ}$ is given by the cross-sections of the horizontal eye equator with the longitudinal body axis, $\psi=0^{\circ}, \theta=90^{\circ}$ is given by the cross-sections of the horizontal eye equator and the vertical body axis, and $\psi=90^{\circ}, \theta=0^{\circ}$ is given by the cross-sections of the horizontal eye equator and the lateral body axis. b Photomontage of an MLG1 neuron as seen in a whole mount preparation after staining the cell with the fluorescent dye Alexa Fluor 488. The dendritic arborisation subtends the upper frontal part of the lobula (right part of the figure), and the axon passes to the contralateral side of the brain (left part of the figure). Scale bar $250 \mu \mathrm{m}$. c Single response of an MLG1 neuron to a bar moving upwards (details see "Materials and methods"). The response is plotted for the entire duration of bar motion $\theta=-10^{\circ}$ and $\theta=60^{\circ}$, and clockwise (i.e. left-to-right) or counter-clockwise (i.e. right-to-left) between $\psi=-40^{\circ}$ and $\psi=40^{\circ}$ along different vertical or horizontal paths within the visual field: the horizontal positions of the three vertical motion traces were $\psi=-20^{\circ}$, $6^{\circ}, 25^{\circ}$ and the vertical positions of the three horizontal motion traces were $\theta=4^{\circ}, 22^{\circ}, 58^{\circ}$. The bar extended over the entire monitor screen and had a size of $80^{\circ} \times$ $5^{\circ}$ (length $\times$ height) when presented horizontally, or $5^{\circ}$ $\times 70^{\circ}$ (length $\times$ height) when presented vertically. The bar either moved upward or downward between $\theta=-10^{\circ}$ and $\theta=60^{\circ}$, and clockwise or counter-clockwise between $\psi=-40^{\circ}$ and $\psi=40^{\circ}$. Artificial stimuli were programmed in MATLAB (The Mathworks, Natick, MA), assigned to the stimulation software, transferred to the image synthesizer and displayed on the monitor (for details see below). Stimulus size and velocity refer to the centre of the screen.

Naturalistic stimuli were presented as a dark spot that moved over the homogenously bright monitor according to the stimulus parameters reconstructed from behavioural data. For technical reasons we had to approximate the form of the target by a black square. Scripts for visual stimulation and data acquisition were written with DT Measure Foundry (Data Translation, Marlboro, MA). Two Data Translation I/O-cards (DT 3001) were used: one for recording neuronal activity and the other for controlling the visual stimulus. Output signals were sent through the analogue channels of one I/O-card to an image synthesizer (Picasso, Innisfree, Cambridge, MA; frame rate: $200 \mathrm{~Hz}$ ) and were displayed on a Tektronix cathode ray tube monitor (Tektronix 608, Tektronix, Wilsonville, OR). The pattern contrast was $82 \%$ (Michelsen contrast) and the mean luminance of the monitor was $31 \mathrm{~cd} / \mathrm{m}^{2}$ measured with a luminance meter (LS 100, Minolta, Osaka, Japan). The control signals of target size and position as well as the membrane potential of the neuron were sampled at a rate of $4 \mathrm{kHz}$ and stored for further analysis.

One run of the visual stimulation protocol consisted of nine different stimuli in a fixed sequence with a pause of $3 \mathrm{~s}$ between each stimulus: five behaviourally generated naturalistic stimuli and four artificial stimuli. The naturalistic stimuli consisted of one reconstructed chase with a fly chasing another fly and of four reconstructed chases with a fly chasing the dummy. The naturalistic stimuli lasted between 300 and 2,500 $\mathrm{ms}$ depending on the duration of the corresponding chase. The duration of artificial stimuli was $1,500 \mathrm{~ms}$. The stimulus protocol was presented repetitively, as long as the intracellular recording was stable (up to $18 \mathrm{~min}$ ). 


\section{Data analysis}

We subtracted the resting potential from each individual response trace and calculated the mean membrane potential over all response traces to each stimulus of each cell. Responses were smoothed using a SavitzkyGolay Filter (span 39, polynomial degree 8; Orfanidis 1996; built-in function of MATLAB-Toolbox) to eliminate high-frequency fluctuations due to noise and active membrane properties. In addition, the mean over the cells that fulfilled our quality criteria (see below) was calculated. For the data analysis of the naturalistic stimuli, the responses were normalised to the maximum amplitude of the potential measured during the stimulations. Data analysis was performed after compensating for the latency between stimulus and response (30 ms).

For motion-sensitive visual neurons, the movement of contrast edges rather than of homogeneous areas is assumed to contribute to the neuronal response. We determined the local motion sensitivity of MLG1 to naturalistic stimuli by analysing the relationship between every single motion step of the stimulus edges and corners-represented by their velocity vectorsand the corresponding membrane potential. The velocity vectors and the corresponding neuronal responses were calculated with a resolution of $5 \mathrm{~ms}$, which is the interframe interval of the stimulus monitor. Since the neuronal responses were sampled at $4 \mathrm{kHz}$, this procedure required time averaging over 20 data points. For further analysis, motion vectors were classified with respect to their velocity and their location in the fly's visual field, and the mean vector and its standard deviation were calculated for three different classes of neuronal responses: weak, moderate and strong (details see "Results"). This procedure allowed us to separate local motion vectors that lead to strong responses from those that do not match the cell's tuning and thus lead to weaker responses.

To quantify the variability of neuronal responses, we calculated the signal-to-noise ratio (SNR) in five MLG1 recordings in different flies. The time-dependent stimulus-induced response component (i.e. signal) was determined as the average of all individual responses across trials. The time-dependent noise component of the responses was determined by calculating the standard deviation of each individual response trace from the ensemble average. The SNR was then obtained as the ratio of the time-dependent signal and the time-dependent noise component. To compare this ratio of MLG1 cells to neurons with well-characterised response properties, we calculated the SNR from recordings of five motion-sensitive HS-cells (Horizontal System cells) (e.g. Hausen 1982a, b) and two motion-sensitive VScells (Vertical System cells) (Hengstenberg 1982; Hengstenberg et al. 1982; Krapp et al. 1998). Although these motion-sensitive cells are thought to be involved in detecting self-motion of the animal, they are known to respond to a single moving spot (Krapp et al. 1998), which allowed us to use the same stimulus for stimulating MLG1 and HS- and VS-cells.

Coherence analysis was used to test a possible representation of specific stimulus parameters in the neuronal response. An integral part of the coherence analysis is the reverse reconstruction technique (Haag and Borst 1998; van Hateren and Snippe 2001): To obtain an estimated (reconstructed) time-dependent stimulus from the measured response, the time-dependent neuronal response is convolved with the linear temporal filter that minimises the difference between the real stimulus and the reconstructed stimulus. The coherence function gives a measure of the similarity between the real and the reconstructed stimulus for different frequencies. The values of the coherence vary between 0 (i.e. both signals are unrelated and/or the system is corrupted by noise) and 1 (i.e. linear relationship). Given a linear system that is corrupted by additive noise, the 'expected coherence' is the coherence between single responses and a noise-free system response. The latter is approximated by averaging many responses and the result can be considered as the response that the best possible (non-linear) model should give (Haag and Borst 1998; van Hateren and Snippe 2001).

\section{Results}

Recordings from 10 MLG1 cells were examined for recording quality (signal strength, signal shape), for systematic drifts in the resting membrane potential and for quality of dye-fill (identification). Most recordings lasted only several minutes. Only five recordings from five different flies lasted sufficiently long for testing the entire stimulation programme while also meeting our recording quality criteria. After dye filling the recorded cells, we identified MLG1 anatomically in a whole mount preparation (Fig. 1b). Since the MLG neurons have already been characterised anatomically before (Gilbert and Strausfeld 1991; Gronenberg and Strausfeld 1991; Strausfeld 1991; Wachenfeld 1994), we refrained from a three-dimensional reconstruction.

\section{Receptive field organisation of MLG1}

To be able to relate the characteristics of MLG1 obtained with naturalistic stimuli to conclusions drawn 
previously, we analysed the activity of this cell type with a sample of experimenter defined ('simple') stimuli similar to those employed more systematically in earlier studies (Gilbert and Strausfeld 1991; Gronenberg and Strausfeld 1991; Wachenfeld, 1994). We did not attempt to replicate these studies in detail, since our results with experimenter defined stimuli qualitatively agreed with the earlier findings. These results will, therefore, be summarised only briefly.

MLG1 neurons respond to motion with a graded shift in membrane potential, often superimposed with spike-like depolarisations, so-called spikelets (Fig. 1c). The membrane potential depolarises by up to $20 \mathrm{mV}$ and spikelets may have amplitudes of up to $25 \mathrm{mV}$. To find out which visual cues induce robust responses in MLG1, we determined the average over all response traces to a given experimenter-defined stimulus and over five cells (see "Materials and methods"). A $30 \mathrm{~ms}$ latency between stimulus and response was estimated as the time delay between the onset of depolarisation in response to motion of a horizontal bar $\left(80^{\circ} \times 5^{\circ}\right)$ moving upwards along the vertical eye axis and the actual onset of bar motion. This value is only a rough estimate because the exact border of the receptive field is unknown. However, $30 \mathrm{~ms}$ corresponds to the value of MLG1 latency obtained in a previous study by measuring the depolarisation onset in response to dot motion stimuli starting at different positions within the receptive field and moving in the four orthogonal directions (Wachenfeld 1994). For the analysis of input-output relations the response traces were shifted by $30 \mathrm{~ms}$ to compensate for the latency between stimulus and response.

In accordance with previous studies (Gilbert and Strausfeld 1991; Strausfeld 1991; Wachenfeld 1994) the receptive field of the MLG1 neuron is located in the dorsofrontal visual field and covers the acute zone of the eye. MLG1 is sensitive to motion of the dot in different directions: Depolarisations to upward motion, i.e. preferred direction motion, are measured nearly all-over the dorsofrontal part of the visual field and are strongest around $\theta=4^{\circ}$ and $\psi=6^{\circ}$. Examples of response averages of MLG1 neurons to experimenterdefined stimuli are shown in Fig. 2. During downward motion (i.e. null-direction motion) the cells are either not depolarised or weakly $(0.5-3 \mathrm{mV})$ hyperpolarised. Furthermore, depolarisations occur for horizontal motion directions, i.e. for clockwise and counterclockwise motion. Looking at the neuronal responses in detail (Fig. 2), we find that the direction selectivity varies within the receptive field: The depolarisations during clockwise motion occur mainly for ipsilaterally presented dots (i.e. $\psi>0^{\circ}$ ), whereas depolarisations
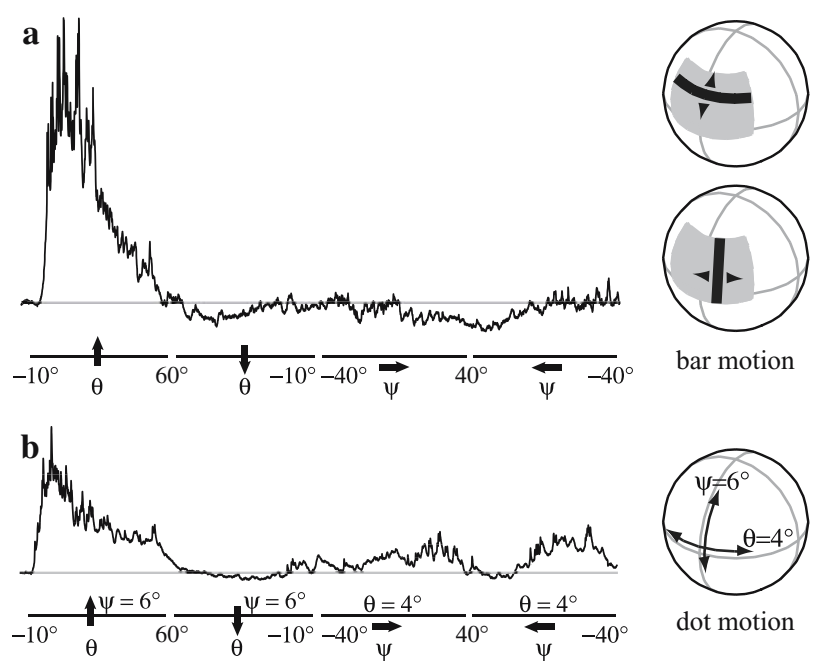

c
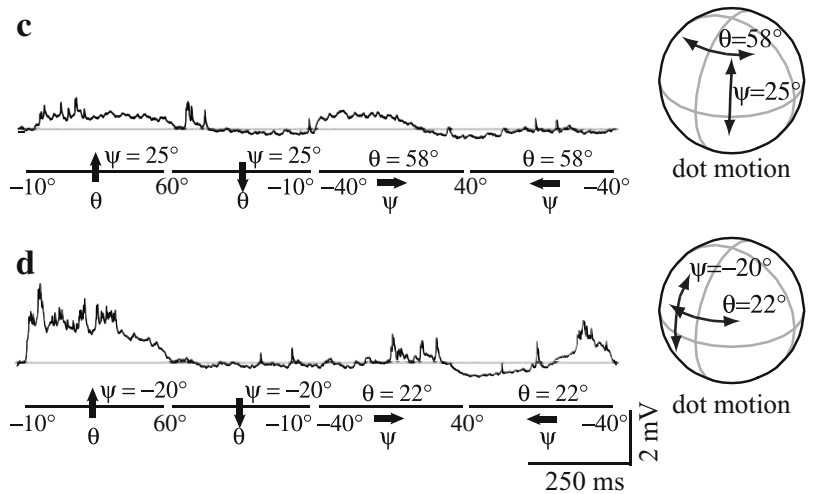

Fig. 2 Responses of MLG1 to experimenter-defined motion stimuli. A spot or a bar was moved with a constant velocity of $180 \%$ s subsequently along different vertical or horizontal tracks. The grey horizontal line denotes the resting potential. Black lines beneath response traces denote the motion duration; the arrow denotes the motion direction (details see "Materials and methods"). a Average of five single response traces to a horizontal and vertical bar moving vertically and horizontally, respectively. b Average of 22 response traces to a spot moving at $\psi=6^{\circ}$ up and down and at $\theta=4^{\circ}$ clockwise and counter-clockwise. c Average of 22 response traces to spots moving up and down at $\psi=25^{\circ}$, clockwise and counter-clockwise at $\theta=58^{\circ}$. d Average of 22 response traces to spots moving up and down at $\psi=-20^{\circ}$, clockwise and counterclockwise at $\theta=22^{\circ}$. Insets: stimulus traces and areas plotted onto the polar coordinate system of the fly's visual field to illustrate the actual positions and extend of stimuli. For clarity, stimuli, stimulus traces and areas are not drawn to scale

for counterclockwise motion occur mainly contralaterally (i.e. $\psi<0^{\circ}$ ). Upward motion of a horizontal bar leads to even larger response amplitudes than upwards moving dots (Fig. 2a). Bars moving downwards evoke, like dots, weak hyperpolarisations. Vertical bars moving horizontally induce, contrary to dots, either no or weak depolarisations (Fig. 2a).

The lacking or only weak hyperpolarisations obtained during null-direction motion are surprising given the pronounced hyperpolarisations during null-direction motion of another class of motion sensitive cells of 
blowflies, the tangential cells (reviews see Hausen and Egelhaaf 1989; Borst and Haag 2002; Egelhaaf et al. 2002; Egelhaaf et al. 2005). Whereas in tangential cells the ratio between depolarisation and hyperpolarisation during preferred and null-direction motion was found in a range 1:0.6 and 1:0.8 (Hausen 1982b; Hengstenberg 1982; Kurtz et al. 2001), the ratio in MLG1 is only 1:0.15.

\section{MLG1 responses to naturalistic stimuli}

To find out to what extent the responses of MLG1 to natural motion stimuli can be explained on the basis of their receptive field properties and direction selectivity as determined by conventional experimenter defined stimuli, we reconstructed what the chasing fly had seen during chasing manoeuvres. Therefore, we reconstructed the trajectories of four male flies chasing after dummy targets that moved on a circular track (two examples are shown in Fig. 3) and of one male fly chasing after another male (trajectory not shown). In the example with a dummy speed of $1.2 \mathrm{~m} / \mathrm{s}$ the chase was terminated with a catch after about $310 \mathrm{~ms}$ (Fig. 3a). With a somewhat faster dummy speed $(1.5 \mathrm{~m} / \mathrm{s})$ the male fly continued to pursue the target for $2.5 \mathrm{~s}$ without succeeding to catch it (Fig. 3b). Because of the limited recording times it proved impracticable to use data from more chases in electrophysiological experiments. It should be noted that the visual input of the chasing fly is more complex when it chases another fly than a dummy: the dummy target moved at constant speed in a horizontal plane, whereas chases of another fly usually have a complex three-dimensional structure. The reduced, but still high complexity of the input during dummy chases facilitated establishing stimulusresponse relationships for behaviourally generated visual stimuli.
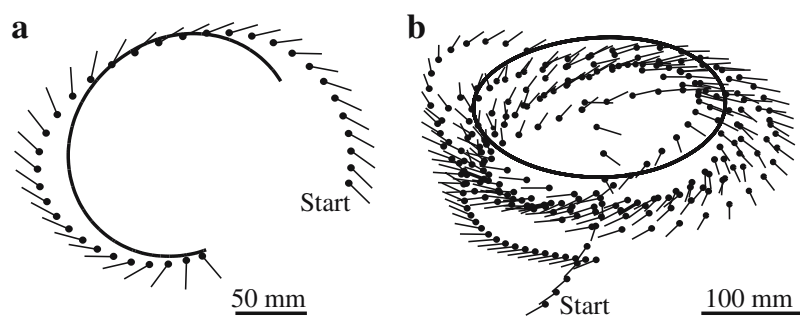

Fig. 3 Reconstruction of three-dimensional flight trajectories of two chasing flights. a The reconstructed flight trajectory of a male fly chasing the target (solid line), which moved on a circular track in top view. The fly is indicated by the position of its body centre (spot) and the orientation of the body length axis (line). The start position of the fly at the beginning of the chase is indicated. The temporal resolution is $20 \mathrm{~ms}$. b Long pursuit of the target without capture in a view from an oblique angle; same plotting conventions as in $\mathbf{a}$. The flight in $\mathbf{a}$ is shown from above; the flight in $\mathbf{b}$ is shown from an oblique direction
At the beginning of the chase, the pursuer is distant and, hence, the size of the dummy is small (Fig. 4a). During the course of the flight the retinal target size increases and tends to be coupled with an increase of the target's vertical position. When the fly draws closer to the target, the retinal image motion will thus contain mainly upward components. The target's vertical and horizontal positions are confined within a restricted range of the fronto-dorsal visual field, as is particularly apparent for long chases (Fig. 4b; see also Boeddeker et al. 2003). In any case, during chasing of a dummy and of real flies, the visual stimuli are characterised by simultaneous variation of several visual parameters over time.

To analyse the responses of MLG1 to naturalistic stimuli, we replayed the reconstructed retinal image sequences on a monitor while simultaneously recording the neuronal activity. When stimulated with these types of naturalistic stimuli, the MLG1 neuron shows strong depolarisations at several instances in time (Fig. 4i, ii). To illustrate the stimulus situation in the chasing fly's visual field, a section of a motion sequence reconstructed from the chase shown in Fig. 3a is projected onto the visual field of the chasing fly (Fig. 5a). In this example, the fly initially approaches the target and both retinal target size and elevation increase, leading to an upward movement of the target. Both, upper and lower edges of the target move in the preferred direction of MLG1 within the most sensitive part of its receptive field. The stimulus-induced membrane potential is depicted by the colour of the respective square. During the displayed stimulus sequence, the cell is subject to strong depolarisation as long as both horizontal edges of the target have a strong upward component (Fig. 5a, see also neuronal response traces Fig. 4i). Note the length of the horizontal edges and the respective high depolarisation level. This is comparable to the abovementioned results with horizontal bars moving upwards inducing maximum response amplitudes (Fig. 2). The response amplitude declines when the edges of the target move mainly horizontally towards the outer parts of the receptive field. This is illustrated in Fig. 5b, which shows a part of the visual input and the corresponding colour coded responses during the chase shown in Fig. 3b: At the beginning, while the horizontal edges of the target move upwards within the most sensitive part of the receptive field, the response amplitude is moderate, but it continually decreases when the stimulus moves downwards and horizontally or towards the outer parts of the receptive field. Given the shortness of the horizontal edges moving upwards, the respective moderate response levels corroborate those measured to a dot moving upwards (Fig. 2). 

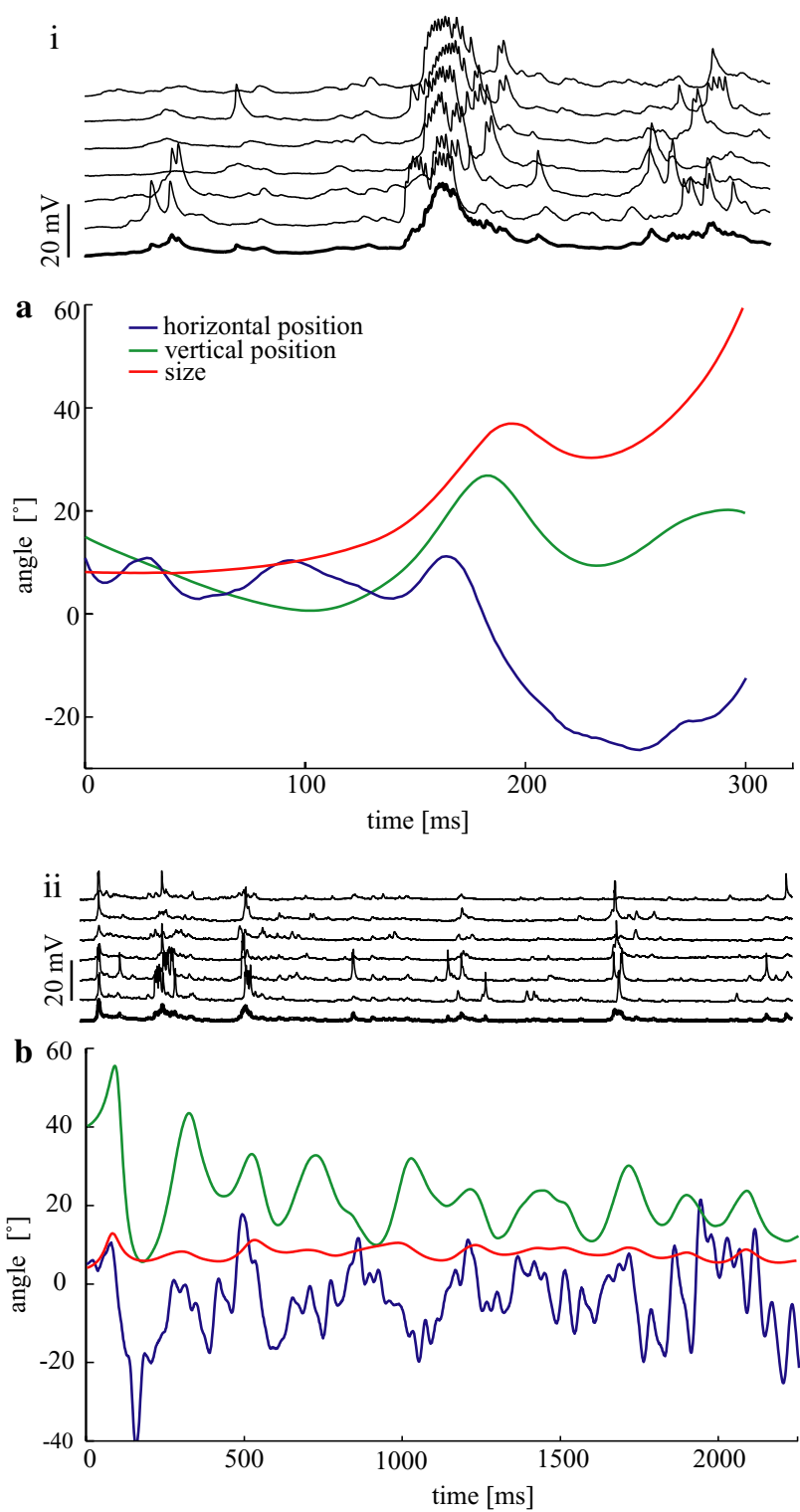

Fig. 4 Reconstructed time-dependent variation of the target's size and position on the retina of the pursuer of two chases and responses of MLG1. a The red line denotes the retinal size, the blue and green line denote the centre of the target's horizontal and vertical position respectively as experienced by the male fly during the chase shown in Fig. 3a. During approach (between about $100-200 \mathrm{~ms}$ and between about $240-300 \mathrm{~ms}$ ) the target's elevation and size tend to increase simultaneously. i Single response traces of MLG1 (thin lines) and the average over the six single traces (bold line, bottom trace) to the stimulus. b Same variables obtained from the chase shown in Fig. 3b. During the chase, the target is well fixated within the acute zone; the target's vertical and horizontal position resides mainly within $\theta=10-30^{\circ}$ and $\psi=-20-20^{\circ}$, which corresponds to the most sensitive part of the receptive field of MLG1 (see also a). ii Single response traces and the average over the six single traces to the stimulus. Note that these are responses from the same cell as shown in $\mathbf{i}$

To quantify the responses to naturalistic visual stimuli and to pool the data of all analysed MLG1 cells we related the response amplitude to the corresponding
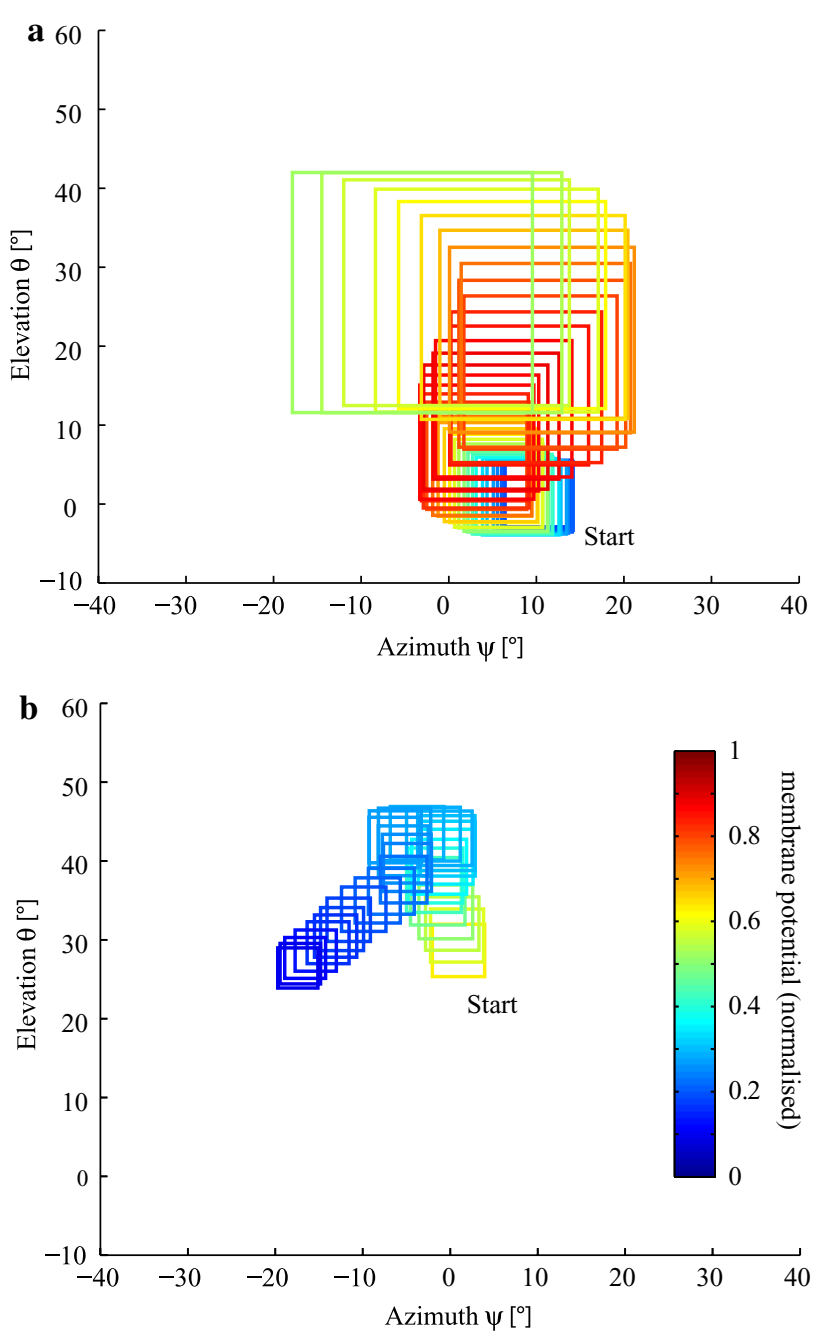

Fig. 5 Time-dependent motion sequences of the stimulus corresponding to two sections of the chases shown in Figs. 3a, b as projected onto the visual field and the corresponding response of the MLG1. For clarity the stimulus (i.e. squared spots) is not filled. a The section shown here corresponds to the time interval between 100 and $180 \mathrm{~ms}$ of Fig. 4a. The amplitude of the normalised membrane potential at any time is denoted by the colour of the respective square (for the details of normalisation, see "Materials and methods"). Warm colours denote strong depolarisations, dark blue denotes strong hyperpolarisation. The size of the square corresponds to the retinal size of the spot at the respective time. The start of the motion sequence is indicated. The temporal resolution is $2.5 \mathrm{~ms}$. b The section shown here corresponds to the time interval between 312 and $430 \mathrm{~ms}$ of Fig. $4 \mathrm{~b}$. The temporal resolution is $3.75 \mathrm{~ms}$. Same plotting conventions as in $\mathbf{a}$

retinal motion vectors by which the cells were driven. This was done by the following procedure. We divided the stimulated visual field of the fly into equally sized grid elements of $14^{\circ} \times 13.3^{\circ}$ (horizontal $\times$ vertical size). Since motion-sensitive neurons respond only to the motion of contrast edges of a stimulus pattern rather than to homogeneous areas, we related the MLG1 responses to the motion vectors of the edges 

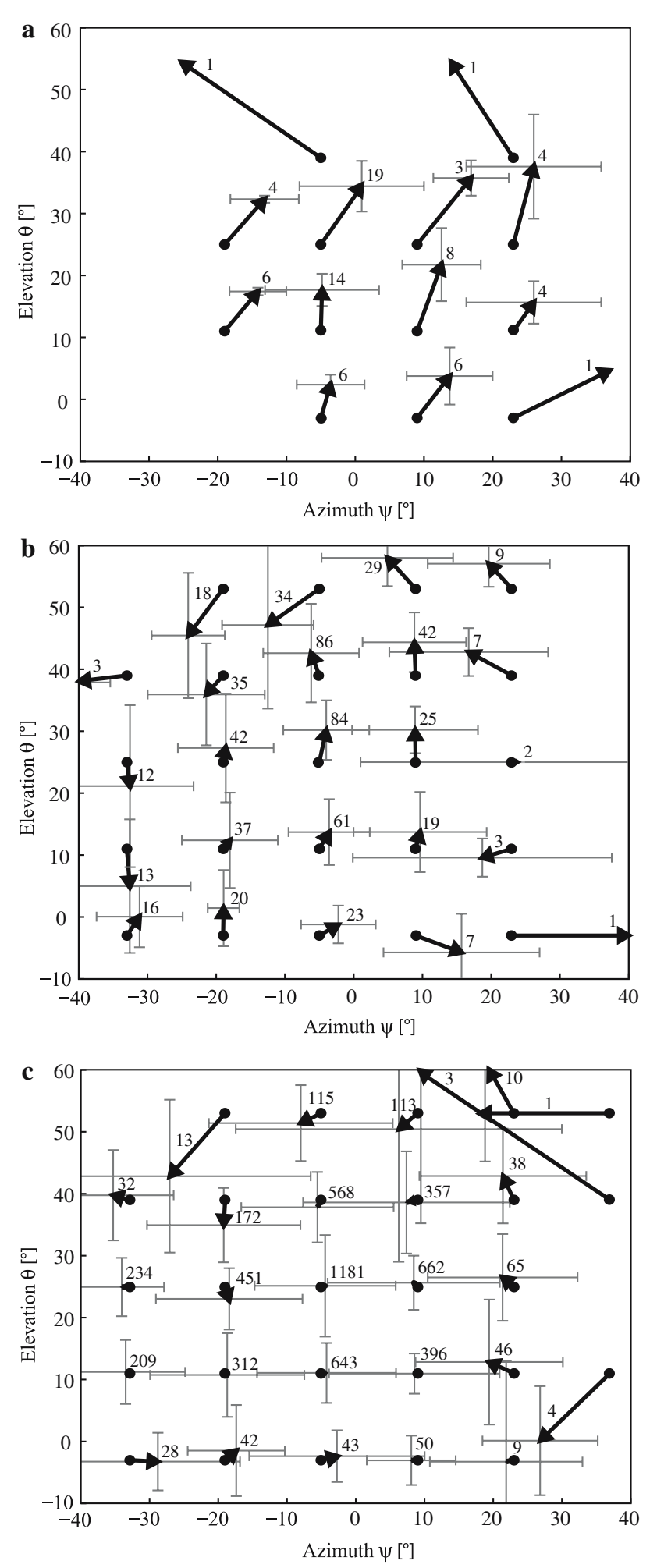

and corners of the target. Therefore, we determined for all naturalistic stimuli which edge motion and/or corner motion occurred within each grid element at each instant of time (time resolution $5 \mathrm{~ms}$ ). The vector length denotes the motion velocity; the vector direction denotes the respective motion direction. To associate
Fig. 6 Average motion vectors corresponding to large a, moderate $\mathbf{b}$ and weak responses $\mathbf{c}$ of MLG1 (for a definition of these response classes, see "Results"). A grid is projected onto the fly's visual field for spatial discretisation. The mean vector that is calculated from the individual motion vectors within each grid element for a given response class (see "Materials and methods") is plotted in the centre of the grid element. The vector length denotes the motion velocity; the vector direction denotes the motion direction. The starting point of the motion vectors corresponds to the centre of the respective grid element. The cross at the vector head denotes the standard deviations of its $x$ - and $y$-components. The number of samples contributing to the mean vector is in most cases depicted in the first quadrant of its standard deviation cross bar. For clarity, arrowheads of short vectors are small. The size of the arrowheads is not of significance. a All mean motion vectors corresponding to large responses include an upward component. b The cell exhibits moderate responses to upward motion within the central part of the receptive field, to horizontal motion and to motion at the outer parts of the receptive field. c For weak responses the corresponding mean motion vectors are short, the number of samples is high. The large standard deviations indicate that individual motion vectors are considerably different in direction and length

the motion vectors with the neuronal response amplitude, the responses were normalised for each cell (see "Materials and methods") and segregated into three response classes, $0.67-1$ for strong, $0.34-0.66$ for moderate and $0-0.33$ for weak responses. This represents an acceptable trade-off between the spatial resolution of the grid and the size of the response classes: a fine grid with small elements would include only a small proportion of the MLG1 responses and thus lead to less significant mean vectors; a coarser grid would blur details of local motion tuning over the neuron's receptive field.

For each grid element, all motion vectors associated with a given response class of MLG1 were determined and the mean vector and the standard deviations of its $x$ - and $y$-components calculated (Fig. 6; numbers denote the number of samples contributing to each mean vector). Large neuronal depolarisations are evoked mainly by motion within a region (Fig. 6a), roughly corresponding to the centre of the receptive field as determined with simple stimuli. This most sensitive area extends from $\theta=0^{\circ}$ to $\theta=40^{\circ}$ vertically and from $\psi=-20^{\circ}$ to $\psi=25^{\circ}$ horizontally. However, large depolarisations occur only when the motion vectors contain an upward component, which is in agreement with the cell's preferred direction, as determined with simple stimuli (Fig. 2). Mean motion vectors that correspond to moderate response amplitudes and that are evoked in the central region have all an upward component. In addition, moderate response amplitudes are measured at the outer parts of the receptive field (Fig. 6b). The majority of the mean velocity vectors corresponding to weak neuronal responses are short 
(Fig. 6c). This results partly from the fact that the individual velocity vectors point into a wide range of directions (see large standard deviations), but may also be due to low motion velocities.

Within the most sensitive region of the cell's receptive field, preferred-direction motion may induce strong, but also moderate or even weak depolarisations. Usually, the responses of motion sensitive neurons depend on stimulus velocity. Which image velocities occur during real chasing situations? We grouped velocities to three classes: low velocities, $0-180^{\circ} / \mathrm{s}$; medium velocities, $180-500^{\circ} / \mathrm{s}$ and high velocities, 500 $2,000 \%$ s. During chases, edge and corner velocities of $180-500 \%$ s occur frequently $(47 \%)$, whereas higher and lower velocities occur in only $29 \%$ and $24 \%$ of cases, respectively. To test whether this feature may affect MLG1 responses, we selected for each response class and each grid element only those responses which were elicited by motion with a strong upward component (i.e. pointing upwards $\theta: 90^{\circ}, \pm 65^{\circ}$ ). The responses were then attributed to one of three velocity ranges (low velocities: $0-180^{\circ} / \mathrm{s}$; medium velocities: $180-500^{\circ} / \mathrm{s}$; high velocities: $500-2,000 \%$ s) and the frequency of occurrence was determined (Table 1). Large responses are only evoked by high and medium velocities, moderate responses predominately by medium velocities, and weak responses almost exclusively by low and medium velocities. These results indicate that during stimulation with naturalistic motion stimuli, the MLG1 neurons exhibit a broad velocity-tuning with preferences for velocities higher than $180^{\circ} / \mathrm{s}$.

There might be another stimulus feature affecting the response amplitude, i.e. the duration of motion in a particular direction. One striking feature of the visual stimuli occurring during chasing manoeuvres is a frequent change in stimulus direction (Fig. 4). An edge of the target moving within the most sensitive part of the receptive field for only 5-10 $\mathrm{ms}$ in the preferred direction may induce a weaker neuronal depolarisation than a target that moves there for $50 \mathrm{~ms}$. Therefore, we

Table 1 Relative frequency of occurrence (in percent) of different response amplitudes for the different classes of motion velocities including the velocity vectors located within the centre of the receptive field and pointing $\theta: 90^{\circ} \pm 65^{\circ}$ upwards

\begin{tabular}{llll}
\hline & $\begin{array}{l}\text { Velocities } \\
0-180 \% \mathrm{~s}\end{array}$ & $\begin{array}{l}\text { Velocities } \\
180-500 \% \mathrm{~s}\end{array}$ & $\begin{array}{l}\text { Velocities } \\
500-2000 \%\end{array}$ \\
\hline Large responses (\%) & 0 & 40 & 60 \\
Moderate responses (\%) & 2.1 & 81.6 & 16.3 \\
Weak responses (\%) & 39.7 & 55.3 & 5 \\
\hline
\end{tabular}

The number of samples for the three velocity classes $(0-180,180$ $500,500-2,000 \%$ s) are 416,844 and 141 , respectively, and for the last line 6427 determined the time intervals within which an edge moved more or less continuously upwards $\left(\theta: 90^{\circ}\right.$, $\left.\pm 65^{\circ}\right)$ within the centre of the receptive field of MLG1 at velocities above $180^{\circ} \mathrm{s}$. The longer an edge moves with a preferred parameter constellation in the receptive field centre, the higher are the evoked response amplitudes (Table 2). In conclusion, the responses of MLG1 to naturalistic chasing stimuli depend on direction, velocity, duration of motion and on the position of motion within the visual field. These results are in accordance with the motion sensitivities and receptive field properties of MLG1 as characterised with simple stimuli.

\section{Variability of MLG1 responses}

The MLG1 neuron is likely to play a role in the extremely fast chasing system. Contrary to expectations that this cell should respond reliably during chasing manoeuvres, the individual responses to the same naturalistic motion sequence show a high degree of variability (Fig. 4i, ii). To put the variability of MLG1 cells into the context of neurons with well-characterised response properties, we recorded motion-sensitive tangential cells (five HS- and two VS-cells) while presenting the same stimulus protocol as was used for characterising MLG1 (data not shown). Although both cell classes are thought to play a role in self-motion detection in both sexes (e.g. review Egelhaaf et al. 2005), they responded quite well to the moving dot-like stimulus. The variability was quantified by comparing the signal-to-noise ratio (for details see "Materials and methods"). For HS-cells, the signal-to-noise ratio is 2.3, for VS-cells 2.0 and for MLG1 neuron 1.7. Thus, the variability among single responses to the same stimulus is slightly higher for MLG1 cells than for VS- and HScells, although the naturalistic stimuli characteristic of

Table 2 Dependence of neuronal response amplitudes on motion duration

\begin{tabular}{lll}
\hline & $\begin{array}{l}\text { Mean motion } \\
\text { duration in preferred } \\
\text { direction }(\mathrm{ms})\end{array}$ & $\begin{array}{l}\text { Standard deviation } \\
\text { of the motion } \\
\text { duration }(\mathrm{ms})\end{array}$ \\
\hline $\begin{array}{l}\text { Large responses } \\
\begin{array}{l}\text { Moderate } \\
\text { responses }\end{array}\end{array}$ & 42.5 & 3.5 \\
$\begin{array}{l}\text { Weak responses } \\
\text { deak }\end{array}$ & 10.9 & 12.9 \\
\hline
\end{tabular}

The mean motion duration gives the average time within which an edge moved with a range of directions centred about upward motion $\left(\theta=90 \pm 65^{\circ}\right)$ in the centre of the receptive field and at velocities $>180^{\circ} / \mathrm{s}$. The membrane potential is assorted into one of three response classes. The number of samples for the class of high, moderate and weak responses is 2,8 and 28 , respectively 
chasing situations that were used in the experiments are not the optimal stimuli of the latter two cell types.

Are retinal target size and position encoded by MLG1?

Previous behavioural experiments led to the conclusion that the chasing control system relies to a large extent on two input parameters: the retinal size and position of the target (Boeddeker et al. 2003; Boeddeker and Egelhaaf 2003, 2005). Therefore, we wanted to test, based on naturalistic stimuli, whether the MLG1 neuron encodes exclusively one of these visual input parameters and calculated the coherence between the neuronal responses and any of these parameters (Fig. 7). The coherence is a frequency-dependent measure that allows us to assess how well the time course of a particular stimulus parameter (here: retinal position or retinal size) can be reconstructed by a linear filter from the neuronal responses (for details, see "Materials and methods"). The coherence varies between 0 (i.e. both signals are unrelated, no reliable reconstruction possible) and 1 (i.e. perfect reconstruction). The coherences between the neuronal responses and the retinal size as well as $x$ - and $y$-position of the target, respectively, are relatively small, indicating that neither of these stimulus parameters is encoded reliably by MLG1. Since a small coherence can have its cause in very noisy neuronal responses as well as in a non-lin-

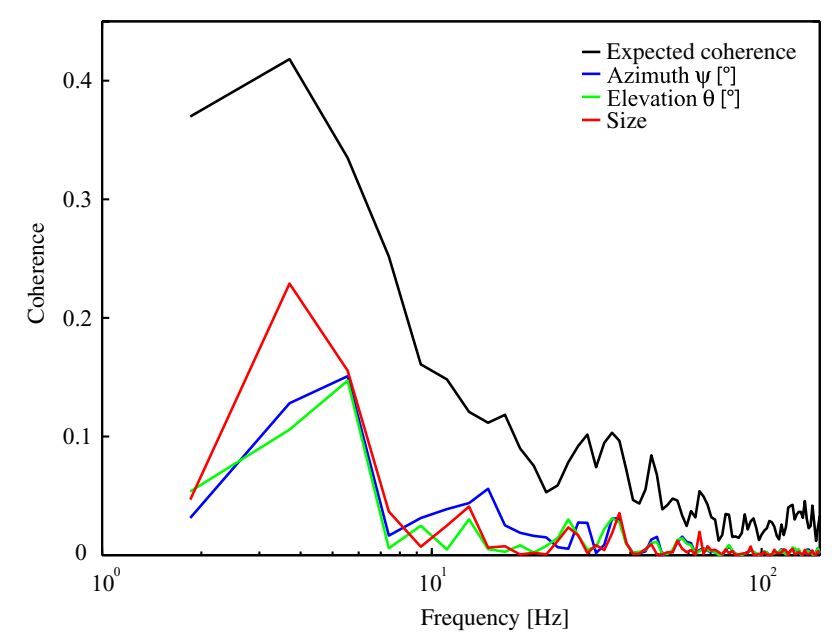

Fig. 7 The coherence between three visual parameters and the neuronal responses as well as the expected coherence are shown. We find small coherences for the retinal size as well as for $x$ - and $y$-position of the target, respectively. Though each of the individual parameters may be somehow represented in the responses of MLG1, we find no explicit linear dependence of the neuronal responses on distinct parameters. The expected coherence can be considered as an upper bound of the coherence function of the system. The deviation of the expected coherence from 1 is induced by noise in the neuronal responses ear relationship between the responses and the analysed stimulus parameter, we also determined the expected coherence. The expected coherence is a measure of neuronal variability and represents the best performance that could, in principle, be obtained for the given noise level of the cell. The expected coherence is considerably smaller than 1 even at low frequencies, corroborating the above conclusion that MLG1 responses reveal a relatively high variability. Moreover, for all tested visual parameters the coherence values are much smaller than the expected coherence. This indicates that each of the individual parameters is somehow represented in the responses of MLG1; however, we have no explicit linear dependence of the neuronal responses on distinct stimulus parameters.

\section{Discussion}

In the present study, we characterised a male-specific visual interneuron (MLG1) of the blowfly Calliphora using naturalistic visual stimuli, as they occur during real chases and tested the hypothesis of a possible representation of specific visual parameters in the neuronal responses.

\section{Characterisation and receptive field properties} of the MLG1 neuron

MLG1 responds with graded shifts in membrane potential, often superimposed by spike-like depolarisations and shows a distinct direction selectivity, which varies within the receptive field. The cell responds best to visual motion stimuli that contain upward components in the dorso-frontal area of the visual field, which is in accordance with previous studies (Gilbert and Strausfeld 1991; Wachenfeld 1994). MLG1 exhibits no pronounced inhibition during null-direction motion which suggests that the mechanism underlying direction selectivity differs in this neuron from the mechanisms of motion detection characterised in tangential cells (e.g. Egelhaaf and Borst 1993). The analyses with naturalistic stimuli reveal the following MLG1 characteristics: (1) sensitivity to several visual parameters (motion direction, position within the visual field, motion velocity and duration of motion) and their variation over time and (2) rather complex response characteristics to the joint occurrence of multiple visual parameters. Because the response characteristics of MLG1 as obtained with naturalistic stimulation corroborate the results found with simple stimuli, pronounced nonlinear interactions of the more complex 
stimulus parameters under natural conditions do not appear to strongly shape MLG1 responses.

MLG1 has a large receptive field that is located in the dorsofrontal region of the retina, thus covering most of the retinal area where the target is fixated during pursuit. MLG1 responds well to stimuli that extend beyond the frontal edge of the visual field of the eyes into the contralateral visual field (Beersma et al. 1977; Wachenfeld 1994). Our findings confirm earlier conclusions that MLG1 does not only receive input from the ipsilateral eye, but also from at least one contralateral neuron. This input may be conveyed to MLG1 by the axon of the contralateral MLG1, since the cell has been suggested to be dye-coupled and thus, possibly electrically coupled with its contralateral counterpart (Gilbert and Strausfeld 1991; Wachenfeld 1994). Additionally, a coupling with the contralateral counterpart could be one way to realise the motion sensitivity of the MLG1 in the other half of the visual system (see Fig. 2).

Male-specific characteristics of the visual system

Chasing behaviour is characterised by extreme virtuosity (Land and Collett 1974; Wehrhahn 1979; Wehrhahn et al. 1982; Zeil 1983; Wagner 1986b, c; Land 1993a, b; Boeddeker et al. 2003). Therefore, the pursuit control system can be assumed to work very fast, efficiently and reliably. The male-specific 'acute zone' has structural and physiological qualities of the visual system which seem to be uniquely adapted for chasing behaviour and should increase the male's ability for successful chasing: Within the acute zone, male Calliphora and Syritta show larger facets and lower interommatidial angles, which are indicative of higher sensitivity and acuity (Land and Eckert 1985; Land 1997). Photoreceptors within an ommatidium are normally differentiated into achromatic (six receptors, i.e. R1-R6) and chromatic (two receptors, i.e. R7 and R8) pathways. However, within the acute zone of male Musca, not only photoreceptors R1-R6, but also R7 and R8, contribute to the achromatic pathway (Franceschini et al. 1981; Hardie et al. 1981). This increased convergence of photoreceptors on second order neurons is believed to improve the detectability of dark targets. Furthermore, male acute zone photoreceptors in Musca respond more strongly than female photoreceptors to moving targets (Burton and Laughlin 2003). Additionally, in Musca, the acute zone photoreceptors show a higher spatial resolution and much faster electrical responses than female photoreceptors, thus allowing encoding higher velocities and smaller targets (Hornstein et al. 2000). Altogether, these characteristics may induce both improvement of the SNR and shortening of the latency of MLGs.

Latency, variability and small-field selectivity of the MLG1 neuron

Although chasing behaviour is extremely fast, the $30 \mathrm{~ms}$ latency of MLG1 estimated in this study is not lower but in the range of other motion sensitive fly neurons, e.g. the H1 neuron (Warzecha and Egelhaaf 2000). This is in accordance with a previous study (Wachenfeld 1994). We did not try to determine the exact latency of the MLG1 neuron, which is very likely to depend on stimulus contrast and on room temperature (Warzecha and Egelhaaf 2000). To test whether the conclusions drawn in the present paper depend on the exact latency value we also tested latencies of 20 and $25 \mathrm{~ms}$. We found that the results are largely independent of the exact latency value because we obtained qualitatively the same results for shorter latencies.

At first sight, a neuronal latency of $30 \mathrm{~ms}$ appears to contradict time lags of $20 \mathrm{~ms}$ as estimated for the control of yaw velocity in chasing behaviour (Boeddeker et al. 2003). However, the behavioural time lag was estimated by cross-correlating the time-dependent angular position of the target and the yaw velocity. In the present study the time lag was estimated by a different method i.e. as the delay between the onset of a bar moving in the preferred direction of the cell and the onset of depolarisation in the neuronal response. Moreover, the temporal resolution in the behavioural study was limited by the interframe interval of $20 \mathrm{~ms}$ and could thus well be slightly shorter or longer than $20 \mathrm{~ms}$. It should be noted that the time lag for the control system of forward velocity is $60-80 \mathrm{~ms}$ and thus larger than that of yaw control and of the MLG1 neuron.

The variability of MLG1 responses is surprising, being not lower, but even slightly higher than the variability of tangential cells stimulated with the same stimuli. Despite this variability, 'optimal' stimuli induce a robust and strong depolarisation of MLG1. Moreover, we cannot exclude that MLG1 operates faster and more reliably than in our electrophysiological experiments when the fly is actually flying and that the responses become more reliable with the age of the fly. For technical reasons, our electrophysiological experiments had to be done on very young flies, i.e. at a age where the males would not yet chase females.

As expected for a neuron presumably tuned to small moving objects, MLG1 responds well to moving spots, but depolarisations induced by a bar moving upwards are stronger than responses to a spot moving in the same direction. This finding is in contrast to a previous 
study in which bars of differing width were used to stimulate MLG1 and the smallest object induced the largest response amplitudes (Wachenfeld 1994). This discrepancy may be due to differences in the details of the stimuli used in the two studies, but can currently not be resolved. However, with regard to horizontal motion, MLG1 responds better to small moving spots than to moving bars. Recently, neurons in the lobula complex of the male hoverfly Eristalis have been characterised as small target motion detectors (STMD). These are sharply tuned to small $\left(0.8^{\circ} \times 3^{\circ}\right)$ moving targets and some STMDs are even inhibited by large objects (Nordström et al. 2006). Neurons tuned to small objects are not specific to insects, but have also been found in the visual system of vertebrates. For instance, directionally selective neurons in the tectofugal system of birds respond to target motion (object diameter $1^{\circ}$ ) and are inhibited by whole-field motion as may occur during self-induced motion (Frost et al. 1990). In this regard the bird neurons are similar to the MLG1 neuron of blowflies and STMD neurons of hoverflies.

\section{The role of MLG1 within a pursuit controller}

What may be the role of MLG1 in visually guided behaviour? Model simulations based on behavioural experiments showed that a control system with retinal size and position as input variables can account for many features of the chasing behaviour of blowflies (Boeddeker and Egelhaaf 2003; Boeddeker et al. 2003). Furthermore, the image velocity of the target was assumed to improve fixation control during chasing (Land and Collett 1974; Land 1992; Boeddeker and Egelhaaf 2005). A possible way to implement this control system may be a neuronal substrate with parallel pathways encoding the target's retinal size, position, and velocity.

From our present knowledge it appears likely that MLG1 takes part in a target fixation control system (Gilbert and Strausfeld 1991) without exclusively signalling target size or position. We employed a coherence analysis, to find out how well retinal target size or target position can be reconstructed on the basis of the neuronal responses. We could not confirm the hypothesis of an explicit representation of either the retinal size or the position of the target in MLG1 responses. Rather, size, position and velocity and their variation over time jointly affect the responses of MLG1.

So far, we have not taken into account the other (at least 11) male specific neurons. It is plausible to assume that a chasing controller may employ the whole ensemble of specified neurons and that these may play different roles in encoding the dynamic visual input during chasing behaviour. The division of the control system into distinct pathways, exclusively signalling separate visual target parameters may thus be convenient for analytical reasons, but seems to get blurred at the neuronal level.

Acknowledgments We are grateful to Roland Kern and Rafael Kurtz for discussion and critical reading, whose comments helped to improve the manuscript, and we thank Amin Hleihil and Roland Kern for programming assistance. This research was supported by the Deutsche Forschungsgemeinschaft (DFG; Graduate Programme 518 'Strategies and Optimisation of Behaviour'). The experiments complied with the current German law and with the 'Principles of animal care', publication No. 86-23, revised 1985 of the National Institute of Health.

\section{References}

Beersma DGM, Stavenga DG, Kuiper JW (1977) Retinal lattice, visual field and binocularities in flies. J Comp Physiol A 119:207-220

Boeddeker N, Egelhaaf M (2003) Steering a virtual blowfly: simulation of visual pursuit. Proc R Soc Lond B 270:1971-1978

Boeddeker N, Kern R, Egelhaaf M (2003) Chasing a dummy target: Smooth pursuit and velocity control in male blowflies. Proc R Soc Lond B 270:393-399

Boeddeker N, Egelhaaf M (2005) A single control system for smooth and saccade-like pursuit in blowflies. J Exp Biol 208:1563-1572

Borst A, Haag J (2002) Neural networks in the cockpit of the fly. J Comp Physiol A 188:419-437

Burton BG, Laughlin SB (2003) Neural images of pursuit targets in the photoreceptor arrays of male and female houseflies Musca domestica. J Exp Biol 206:3963-3977

Collett TS, Land MF (1975) Visual control of flight behaviour in the hoverfly Syritta pipiens L. J Comp Physiol A 99:1-66

Collett TS (1980) Angular tracking and the optomotor response. An analysis of visual reflex interaction in a hoverfly. J Comp Physiol A 140:145-158

Egelhaaf M, Borst A (1993) A look into the cockpit of the fly: visual orientation, algorithms, and identified neurons. J Neurosci 13(11):4563-4574

Egelhaaf M, Kern R, Krapp HG, Kretzberg J, Kurtz R, Warzecha AK (2002) Neural encoding of behaviourally relevant visualmotion information in the fly. J Neurosci 25:96-102

Egelhaaf M, Grewe J, Karmeier K, Kern R, Kurtz R, Warzecha AK (2005) Novel approaches to visual information processing in insects: case studies on neuronal computations in the blowfly. In: Christensen TA (ed) Methods in insect sensory neuroscience. CRC Press, Boca Raton, pp 185-212

Franceschini N, Kirschfeld K (1971) Pseudopupil phenomena in the Drosophila compound eye. Kybernetik 9:159-182

Franceschini N, Hardie R, Ribi W, Kirschfeld K (1981) Sexual dimorphism in a photoreceptor. Nature 291:241-244

Frost BJ, Wylie DR, Wang YC (1990) The processing of object and self-motion in the tectofugal and accessory optic pathways of birds. Vis Res 30:1677-1688

Gilbert C, Strausfeld NJ (1991) The functional organisation of male-specific visual neurons in flies. J Comp Physiol A 169:395-411

Gronenberg W, Strausfeld NJ (1991) Descending pathways connecting the male-specific visual system of flies to the neck and flight motor. J Comp Physiol A 169:413-426 
Haag J, Borst A (1998) Active membrane properties and signal encoding in graded potential neurons. $J$ Neurosci 18(19):7972-7986

Hardie RC, Franceschini N, Ribi W, Kirschfeld K (1981) Distribution and properties of sex-specific photoreceptors in the fly Musca domestica. J Comp Physiol A 145:139-152

Hausen K (1982a) Motion sensitive interneurons in the optomotor system of the fly. I The horizontal cells: structure and signals. Biol Cybern 45:143-156

Hausen K (1982b) Motion sensitive interneurons in the optomotor system of the fly. II The horizontal cells: receptive field organization and response characteristics. Biol Cybern 46:67-79

Hausen K, Strausfeld NJ (1980) Sexually dimorphic interneuron arrangements in the fly visual system. Proc R Soc Lond B 208:57-71

Hausen K, Egelhaaf M (1989) Neural mechanisms of visual course control in insects. In: Stavenga DG, Hardie RC (eds) Facets of vision. Springer, Heidelberg, pp 391-424

Hengstenberg R (1982) Common visual response properties of giant vertical cells in the lobula plate of the blowfly Calliphora. J Comp Physiol A 149:179-193

Hengstenberg R (1993) Multisensory control in insect oculomotor systems. In: Miles FA, Wallmann J (eds) Visual motion and its role in the stabilisation of gaze. Elsevier, Amsterdam, pp 285-298

Hengstenberg R, Hausen K, Hengstenberg B (1982) The number and structure of giant vertical cells (VS) in the lobula plate of the blowfly Calliphora erythrocephala. J Comp Physiol A 149:163-177

Hornstein EP, O'Carrol DC, Anderson JC, Laughlin SB (2000) Sexual dimorphism matches photoreceptor performance to behavioural requirements. Proc R Soc Lond B 267:21112117

Hüls T (2005) Instability helps virtual flies to mate. Biol Cybern 93:222-229

Kayser C, Körding KP, König P (2004) Processing of complex stimuli and natural scenes in the visual cortex. Curr Opin Neurobiol 14:468-473

Kern R, van Hateren JH, Michaelis C, Lindemann JP, Egelhaaf $M$ (2005) Function of a fly motion-sensitive neuron matches eye movements during free flight. PloS Biol 3(6):e171

Krapp HG, Hengstenberg B, Hengstenberg R (1998) Dendritic structure and receptive-field organization of optic flow processing interneurons in the fly. J Neurophysiol 79:1902-1917

Kurtz R, Warzecha AK, Egelhaaf M (2001) Transfer of visual motion information via graded synapses operates linearly in the natural activity range. J Neurosci 21(17):6957-6966

Land MF (1973) Head movement of flies during visually guided flight. Nature 243:299-300

Land MF (1992) Visual tracking and pursuit: Humans and arthropods compared. J Insect Physiol 38:939-951

Land MF (1993a) The visual control of courtship behaviour in the fly Poecilobothrus nobilitatus. J Comp Physiol A 173:595-603

Land MF (1993b) Chasing and pursuit in the dolichopodid fly Poecilobothrus nobilitatus. J Comp Physiol A 173:605-613

Land MF (1997) Visual acuity in insects. Annu Rev Entomol 42:147-177

Land MF, Collett TS (1974) Chasing behaviour of house flies (Fannia canicularis). J Comp Physiol A 89:331-357
Land MF, Eckert H (1985) Maps of the acute zones of fly eyes. J Comp Physiol A 156:525-538

Lindemann JP, Kern R, Michaelis C, Meyer P, van Hateren JP, Egelhaaf M (2003) FliMax, a novel stimulus device for panoramic and highspeed presentation of behaviourally generated optic flow. Vis Res 43:779-791

Nordström K, Barnett PD, O’Carroll DC (2006) Insect detection of small targets moving in visual clutter. PLoS Biol 4(3):e54

Orfanidis SJ (1996) Introduction to signal processing. PrenticeHall, Englewood Cliffs

Reinagel P (2001) The many faces of adaptation. Nature 412:776777

Schilstra C, van Hateren JH (1998) Using miniature sensor coils for simultaneous measurement of orientation and position of small, fast-moving animals. J Neurosci Methods 83:125-131

Simoncelli EP (2003) Vision and the statistics of the visual environment. Curr Opin Neurobiol 13:144-149

Simoncelli EP, Olshausen BA (2001) Natural image statistics and neural representation. Annu Rev Neurosci 24:1193-1216

Strausfeld NJ (1991) Structural organisation of male-specific visual neurons in calliphorid optic lobes. J Comp Physiol A 169:379-393

van Hateren JH (1997) Processing of natural time series of intensities by the visual system of the blowfly. Vis Res 37:34073416

van Hateren JH, Schilstra C (1999) Blowfly flight and optic flow. II. Head movements during flight. J Exp Biol 202:1491-1500

van Hateren JH, Snippe HP (2001) Information theoretical evaluation of parametric models of gain control in blowfly photoreceptor cells. Vision Research 41:1851-1865

van Hateren JH, Kern R, Schwerdtfeger G, Egelhaaf M (2005) Function and coding in the blowfly $\mathrm{H} 1$ neuron during naturalistic optic flow. J Neurosci 25:4343-4352

Wachenfeld A (1994) Elektrophysiologische Untersuchungen und funktionelle Charakterisierung männchenspezifischer visueller Interneurone in der Schmeißfliege Calliphora erythrocephala (Meig.). Doctoral dissertation, Universität Köln

Wagner H (1986a) Flight performance and visual control of flight of the free-flying housefly (Musca domestica L.) I. Organisation of the flight motor. Philos Trans R Soc Lond B 312:527551

Wagner H (1986b) Flight performance and visual control of flight of the free-flying housefly (Musca domestica L.) II. Pursuit of targets. Philos Trans R Soc Lond B 312:553-579

Wagner H (1986c) Flight performance and visual control of flight of the free-flying housefly (Musca domestica L.) III. Interactions between angular movement induced by wide- and smallfield stimuli. Philos Trans R Soc Lond B 312:581-595

Warzecha AK, Egelhaaf M (2000) Response latency of a motionsensitive neuron in the fly visual system: dependence on stimulus parameters and physiological conditions. Vis Res 40:2973-2983

Wehrhahn C (1979) Sex-specific differences in the chasing behaviour of houseflies (Musca). Biol Cybern 32:239-241

Wehrhahn C, Poggio T, Buelthoff H (1982) Tracking and chasing in houseflies (Musca) An analysis of 3-D flight trajectories. Biol Cybern 45:123-130

Zeil J (1983) Sexual dimorphism in the visual system of flies: the free flight behaviour of male Bibionidae (Diptera). J Comp Physiol A 150:395-412 